CERN-PH-TH/2006-159

CPHT-RR-nnn.0806

LPT-ORSAY-06-55

hep-th/0608054

\title{
Dual realizations of dynamical symmetry breaking
}

\author{
Emilian Dudas ${ }^{a, b, c}$ and Chloé Papineau ${ }^{c, b}$ \\ ${ }^{a}$ CERN Theory Division, CH-1211, Geneva 23, Switzerland \\ ${ }^{b}$ Centre de Physique Théorique ${ }^{1}$, Ecole Polytechnique, 91128 Palaiseau Cedex, France \\ ${ }^{c}$ LPT $^{2}$, Bât. 210, Univ. de Paris-Sud, 91405 Orsay Cedex, France \\ Emilian.Dudas@cpht.polytechnique.fr, chloe.papineau@th.u-psud.fr
}

\begin{abstract}
We show the infrared equivalence between a recently proposed model containing a six dimensional scalar field with a four-dimensional localized Higgs type potential and the four-dimensional Nambu-Jona-Lasinio (NJL) model. In the dual NJL description, the fermions are localized at the origin of a large two-dimensional compact space. Due to a classical running effect above the compactification scale, the four-fermion coupling of the NJL model increases from the cutoff scale down to the compactification scale, providing the large Fermi coupling needed for the dynamical symmetry breaking. We also present a string theory embedding of our field-theory construction. On more general grounds, our results suggest that $4 \mathrm{~d}$ models with dynamical symmetry breaking can be given a higher dimensional description in terms of field theories with nontrivial boundary conditions in the internal space.
\end{abstract}

\footnotetext{
${ }^{1}$ Unité mixte du CNRS et de l'EP, UMR 7644.

${ }^{2}$ Unité mixte du CNRS, UMR 8627.
} 


\section{Introduction and Conclusions}

Dimensional transmutation and generation of a small scale is a remarkable result common to many quantum field theories, most notably the four-dimensional QCD and the twodimensional Gross-Neveu model. The effect is also realized in two-dimensional quantum mechanics with a deep (delta-like) attractive potential and in six-dimensional scalar models ${ }^{3}$ with $4 \mathrm{~d}$ localized scalar potential and a large but compact transverse space. The last example, put forward in [2, was analyzed from the point of the quantum-mechanical problem in the case of a perturbative coupling $\mu$ appearing as the (dimensionless) localized parameter interpreted as a mass term in $4 \mathrm{~d}$. It was shown that for a $6 \mathrm{~d}$ scalar field with Dirichlet boundary condition on a large two-dimensional compact space taken for simplicity to be a disk, there is a phase transition with a very light (compared to the compactification scale) particle for a small critical value $\mu_{c} \ll 1$. The parameter $\mu$ was shown to run between the cutoff scale $\Lambda$ and the compactification scale $R^{-1}$, such that precisely at the critical point, $\mu$ becomes large at $R^{-1}$. A very similar phenomenon of appearance of a light state close to a critical point where an (four-fermion) interaction becomes strong is in the $4 \mathrm{~d}$ Nambu-JonaLasinio (NJL) model [3]. The purpose of the present paper is to study closer this analogy and argue that the 6d model studied in [2] and the 4d NJL model are, in a sense which will be defined in detail later on, dual descriptions of the same physics. Our starting point is to provide an explicit framework in which the $4 \mathrm{~d}$ localized potential is generated, by a Yukawa interaction of the bulk scalar field with $\mathrm{N} 4 \mathrm{~d}$ localized fermions. In the large $\mathrm{N}$ limit, integrating out the fermions produces precisely the potential needed for the symmetry breaking. Alternatively, we show that integrating out the bulk scalar leads to a dual 4d NJL model with chiral symmetry breaking, where the Fermi coupling is generated at tree-level by scalar bulk exchange. We show that the critical Fermi coupling calculated by NJL methods in the large $\mathrm{N}$ limit agrees with the bulk $6 \mathrm{~d}$ calculation of the critical coupling calculated as a problem with nontrivial boundary conditions. The $6 \mathrm{~d} \leftrightarrow 4 \mathrm{~d}$ duality we study exchanges some quantum and classical natures of the symmetry breaking phenomenon. In the bulk $6 \mathrm{~d}$ picture, the quantum (Yukawa) interactions are completely encoded in a boundary condition, the localized scalar potential, whereas the symmetry breaking can be studied as a quantummechanical problem with nontrivial boundary condition and can be understood as a result of a classical running effect in the transverse $2 \mathrm{~d}$ space. In the $4 \mathrm{~d}$ NJL picture, the symmetry breaking is provided by the nonperturbative self-consistent gap equation [3, 4, 5], but in addition the four-fermion coupling has a classical logarithmic running between the cutoff and the compactification scale. When in the bulk picture $\mu=\mu_{c}$ at $\Lambda$, the four-fermion coupling in the NJL picture at the compactification scale reaches the critical value for the dynamical symmetry breaking $G\left(R^{-1}\right)=G_{c}$. Our main interest in this equivalence is that, whereas a consistent treatment of the NJL model involves nonperturbative techniques like the large $N$ expansion or going below $4 \mathrm{~d}$ and using UV nontrivial fixed points and $1 / \epsilon$ techniques, the bulk analysis is essentially classical ${ }^{4}$ and does not need, in principle, any nonperturbative

\footnotetext{
${ }^{3}$ For gravitational aspects of codimension two models, see e.g. [1].

${ }^{4}$ Throughout the paper by "classical" we mean classical from the point of view of quantum field theory, i.e. no quantum interaction. The treatment is still quantum mechanical.
} 
techniques.

The structure of the paper is as follows. In section 2 we review the six dimensional model worked out in [2] and argue that, in addition to the perturbative critical coupling $\mu_{c}$ we found there, there are other critical points corresponding to large values $\mu_{c}^{(n)}>1$. In section 2.1 we study a similar setup in which the nontrivial boundary condition in the compact space is replaced by a bulk mass and Neumann boundary condition. In Section 3 we define the 6d model on an orbifold space instead of a disk, which is more suitable for a microscopic (string theory) realization. Section 4 contains the main arguments concerning the infrared equivalence between the $6 \mathrm{~d}$ scalar model with nontrivial boundary condition and the $4 \mathrm{~d}$ NJL model. Section 5 generalizes the previous section to a (softly broken) supersymmetric theory. Section 6 provides an explicit string theory realization of the present setup in an orientifold of type IIB strings with D-branes.

\section{Six dimensional phase transition : perturbative and nonperturbative critical couplings}

Recently, 2] addressed the problem of spontaneous symmetry breaking in a $6 \mathrm{~d}$ scalar model with 4d localized Higgs potential. The corresponding action reads ${ }^{5}$ :

$$
\begin{aligned}
& S=\int d^{4} x d^{2} y\left[\frac{1}{2}\left(\partial_{M} \phi\right)^{2}-V_{\delta}(\phi)\right], \\
& V_{\delta}(\phi)=\left(-\frac{\mu^{2}}{2} \phi^{2}+\frac{\lambda}{4} \phi^{4}\right) \cdot \delta^{2}(y) .
\end{aligned}
$$

The scalar field $\phi$ has dimension two and therefore $\mu^{2}$ is dimensionless. The scalar potential is localized at the origin of the compact space. We resolve the singularity at $y=0$ by introducing a disk $r<\epsilon$ supporting the potential,

$$
\begin{array}{ll}
V(\phi)=\frac{1}{\pi \epsilon^{2}}\left(-\frac{\mu^{2}}{2} \phi^{2}+\frac{\lambda}{4} \phi^{4}\right) & \text { for } 0<r<\epsilon, \\
V(\phi)=0 & \text { for } \epsilon<r<R .
\end{array}
$$

According to [6, 7, 2, there is a "classical" running of the tachyonic mass parameter

$$
\frac{1}{\mu^{2}(Q)}=\frac{1}{\mu^{2}(\Lambda)}-\frac{1}{2 \pi} \ln \left(\frac{\Lambda}{Q}\right) \text {. }
$$

It was shown in 2] for a compact two-dimensional space, chosen to be a disk of radius $R$, with 4d localized Higgs potential and Dirichlet boundary condition

$$
\left.\phi\right|_{r=R}=0
$$

\footnotetext{
${ }^{5}$ We are using a $(+,-,-,-,-,-)$ metric. The index $M$ denotes bulk coordinates and runs from $0,1,2,3,4,5$, while $\mu=0,1,2,3$ denotes brane coordinates. We'll use either $x^{4,5}$ or $y_{1,2}$ to denote the two extra dimensions.
} 
that this model has a phase transition for a small critical value

$$
\frac{\mu_{c}^{2}}{2 \pi} \ln (R \Lambda)=1
$$

where $\Lambda$ is a UV cutoff defined in connection with the resolution of the delta singularity $\Lambda=$ $1 / \epsilon$. So the phase transition happens precisely when the renormalized value $\mu_{c}^{2}\left(R^{-1}\right) \rightarrow \infty$ blows up at the compactification scale. The running interpretation breaks down close to the phase transition point. The classical running of $\mu$ induces also a running for the self-coupling $\lambda$, according to the $\mathrm{RG}$ equation

$$
Q \frac{d \lambda}{d Q}=-\frac{2}{\pi} \mu^{2} \lambda
$$

which, by using (3), readily integrates to

$$
\lambda(Q)=\frac{\lambda(\Lambda)}{\left(1-\frac{\mu^{2}}{2 \pi} \ln \frac{\Lambda}{Q}\right)^{4}} .
$$

Notice that at the phase transition point $\mu=\mu_{c}$,

$$
\mu\left(R^{-1}\right) \rightarrow \infty \quad, \quad \lambda\left(R^{-1}\right) \rightarrow \infty
$$

We will argue later on in section 4 that in a dual $4 \mathrm{~d}$ theory which turns out to be a NJL theory, the conditions (8) have the interpretation of compositeness conditions of [5]. Close to the critical coupling, however, the running interpretation breaks down and actually the higher-dimensional $6 \mathrm{~d}$ and also the $4 \mathrm{~d}$ physics turn out to be perturbative.

We now review and slightly update the arguments of [2] by arguing that there are actually additional but large critical couplings $\mu_{c}^{(n)} \geq \sqrt{4 \pi}$, defined by the presence of a $4 \mathrm{~d}$ massless mode in the spectrum. Assuming that this exists, slightly below it $\mu \leq \mu_{c}$, in the background $\phi_{c}=0$, the field eqs. for a $4 \mathrm{~d}$ mode of mass $M^{2}=p^{2}$, are

$$
\begin{aligned}
& \Delta^{(2)} \phi+\frac{\mu^{2}}{\pi \epsilon^{2}} \phi=0, \quad r<\epsilon, \\
& \Delta^{(2)} \phi+p^{2} \phi=0, \quad r>\epsilon,
\end{aligned}
$$

where we neglected the mass $p^{2}$ inside the brane (this is a very good approximation for all masses much lighter than the cutoff $\Lambda=1 / \epsilon$ ). The solutions of (91) with Dirichlet boundary condition (41) and for $p^{2} \ll R^{-2}$ are

$$
\begin{aligned}
& \phi(r)=f_{0} J_{0}\left(\frac{\mu r}{\sqrt{\pi} \epsilon}\right), \quad r<\epsilon, \\
& \phi(r)=a\left[\ln \frac{R}{r}-\frac{p^{2} r^{2}}{4} \ln \frac{R}{r}+\frac{p^{2}}{4}\left(R^{2}-r^{2}\right)\right], \quad r>\epsilon .
\end{aligned}
$$


The zero mass solutions $p^{2}=0$ define the whole set of critical couplings. The matching conditions of the logarithmic derivative of the wave function at $r=\epsilon$ then give

$$
\frac{\mu_{c} J_{0}^{\prime}\left(\frac{\mu_{c}}{\sqrt{\pi}}\right)}{\sqrt{\pi} J_{0}\left(\frac{\mu_{c}}{\sqrt{\pi}}\right)} \ln \frac{R}{\epsilon}=-1 .
$$

For small $\mu_{c}$, eq. (11) has the unique solution (15), which is indeed small provided that $R^{-1} \ll \Lambda$. Equation (11), however, has an infinite discrete set of solutions, as can easily be shown by a numerical plot. The peculiarity of the perturbative solution (5) is that the wave function (10) inside the brane $r<\epsilon$, and actually also the wave functions of the massive modes below the cutoff $\Lambda$, are almost constant. On the contrary, the wavefunctions corresponding to the "nonperturbative" critical couplings (in the sense $\mu_{c}^{2} / 4 \pi>1$ ) have substantial variation inside the brane. Slightly below the critical coupling $(\mathrm{s}) \mu_{c}$, the zero mode becomes massive, with a mass $M$ given approximately by

$$
M^{2}=p_{\mu} p^{\mu} \simeq \frac{2\left(\mu_{c}^{2}-\mu^{2}\right)}{\pi R^{2}}\left(\ln \frac{R}{\epsilon}\right)^{2} .
$$

The mass (12) reduces to the one computed in [2] in the case of the perturbative critical coupling (5). Close to the other critical couplings $\mu \simeq \mu_{c}^{(n)}$ the light mode can also be described in a $4 \mathrm{~d}$ effective field theory. The $6 \mathrm{~d}$ field is decomposed according to $\phi(x, r)=$ $\sigma(x) \chi(r)$, where

$$
\begin{aligned}
& \chi(r)=\sqrt{\frac{2}{\pi R^{2}}} \ln \left(\frac{R}{\epsilon}\right) \frac{J_{0}\left(\frac{\mu r}{\sqrt{\pi}}\right)}{J_{0}\left(\frac{\mu}{\sqrt{\pi}}\right)}, \quad r<\epsilon, \\
& \chi(r)=\sqrt{\frac{2}{\pi R^{2}}} \ln \frac{R}{r}, \quad r>\epsilon .
\end{aligned}
$$

The effective $4 \mathrm{~d}$ potential for $\sigma$ is given by

$$
V_{e f f}(\sigma)=\frac{m_{4}^{2}}{2} \sigma^{2}+\frac{\lambda_{4}}{4} \sigma^{4}
$$

where the mass parameter and the coupling are given by

$$
\begin{aligned}
& m_{4}^{2}=-\frac{2 \mu^{2}}{\epsilon^{2}} \int_{0}^{\epsilon} r d r \chi^{2}+\frac{2 \mu^{2}}{\epsilon^{2}} \int_{0}^{\epsilon} r d r\left(\chi^{\prime}\right)^{2}+2 \pi \int_{\epsilon}^{R} r d r\left(\chi^{\prime}\right)^{2} \\
& \lambda_{4}=\frac{2 \lambda}{\epsilon^{2}} \int_{0}^{\epsilon} r d r \chi^{4}(r)=\frac{8 \lambda}{\pi^{2} \mu^{2} R^{4}} \frac{1}{J_{0}^{4}(\mu / \sqrt{\pi})}\left(\ln \frac{R}{\epsilon}\right)^{4} \int_{0}^{\frac{\mu}{\sqrt{\pi}}} x d x J_{0}^{4}(x),
\end{aligned}
$$

where the derivative in $\chi^{\prime}$ is wrt the argument of the Bessel functions. Very close to $\mu_{c}^{(n)}$, the resulting mass coincides with $M^{2}$ in (12), showing the validity of the $4 \mathrm{~d}$ description. The presence of light $4 \mathrm{~d}$ modes close to the large critical couplings $\mu_{c}^{(n)}$ is a signature of a UV-IR mixing, where the UV physics changes the masses in the IR. While in a microscopic 
theory in which $\mu$ is generated dynamically, large values ask presumably for nonperturbative effects, from the bulk $2 \mathrm{~d}$ viewpoint, $\mu$ changes only the boundary conditions of the scalar field and its consequences can be treated exactly quantum-mechanically. On the other hand, the explicit values of $\mu_{c}^{(n)}$ depend on the way we regularize the origin of the $2 \mathrm{~d}$ space and thus on the UV physics. Therefore, the physical consequences of the large critical couplings are probably highly sensitive on the UV physics. This is not the case for the small critical coupling (5), whose value is insensitive to the regularization procedure and therefore of the UV physics, as we explicitly check by using a different regularization in section 3 .

\subsection{Phase transition with bulk mass and boundary Higgs potential}

A natural question arising in the present setup is what happens if one replaces the positive contribution to the mass coming from the Dirichlet boundary condition by a bulk mass $m$, keeping the $4 \mathrm{~d}$ localized Higgs-type potential. The action describing this case is given by

$$
\begin{aligned}
& S=\int d^{4} x d^{2} y\left[\frac{1}{2}\left(\partial_{M} \phi\right)^{2}-\frac{1}{2} m^{2} \phi^{2}-V_{\delta}(\phi)\right] \\
& V_{\delta}(\phi)=\left(-\frac{\mu^{2}}{2} \phi^{2}+\frac{\lambda}{4} \phi^{4}\right) \cdot \delta^{2}(y)
\end{aligned}
$$

where the field $\phi$ has now Neumann boundary condition

$$
\left.\partial_{r} \phi\right|_{r=R}=0
$$

We are working in the unbroken phase $\phi_{c}=0$, in which case the field equations for a $4 \mathrm{~d}$ field of mass $p^{2}$ are

$$
\begin{aligned}
& \Delta^{(2)} \phi+\frac{\mu^{2}}{\pi \epsilon^{2}} \phi=0, \quad r<\epsilon, \\
& \Delta^{(2)} \phi+\left(p^{2}-m^{2}\right) \phi=0, \quad r>\epsilon .
\end{aligned}
$$

By defining $q^{2}=m^{2}-p^{2}$, we find the solutions of (18) with Neumann boundary conditions (17) and for $q^{2} \ll R^{-2}$ to be

$$
\begin{aligned}
& \phi(r)=f_{0} J_{0}\left(\frac{\mu r}{\sqrt{\pi} \epsilon}\right), \quad r<\epsilon, \\
& \phi(r)=a\left[1+\frac{q^{2} R^{2}}{2} \ln \frac{R}{r}+\frac{q^{2}}{4} r^{2}\right], \quad r>\epsilon .
\end{aligned}
$$

Matching conditions at $r=\epsilon$ for zero mass solutions $p^{2}=0$ define the critical couplings in this case to be given by the solutions of

$$
\left[1-\frac{\mu_{c} J_{1}\left(\frac{\mu_{c}}{\sqrt{\pi}}\right)}{\sqrt{\pi} J_{0}\left(\frac{\mu_{c}}{\sqrt{\pi}}\right)} \ln \frac{R}{\epsilon}\right] \frac{m^{2} R^{2}}{2}=\frac{\mu_{c} J_{1}\left(\frac{\mu_{c}}{\sqrt{\pi}}\right)}{\sqrt{\pi} J_{0}\left(\frac{\mu_{c}}{\sqrt{\pi}}\right)} .
$$


Analogously to the case discussed in the previous section, eq. (20) has an infinity but discrete number of solutions $\mu_{c}^{(n)}$, out of which only one is perturbative $\mu_{c} \ll 1$. In this perturbative case, similarly to (5), there is a classical running interpretation of the critical coupling

$$
\pi R^{2} m^{2}=\frac{\mu_{c}^{2}}{1-\frac{\mu_{c}^{2}}{2 \pi} \ln \frac{R}{\epsilon}}=\mu_{c}^{2}\left(R^{-1}\right)
$$

where $\mu_{c}^{2}\left(R^{-1}\right)$ is the renormalized value of the (perturbative) critical coupling at the compactification scale $Q=R^{-1}$. From (21) it follows that for small bulk mass $m^{2} R^{2} \ll 1, \mu$ stays perturbative at all energies above the compactification scale, whereas for large masses $m^{2} R^{2} \geq 1, \mu$ enters strong coupling regime if there is a light $4 \mathrm{~d}$ mode in the spectrum.

Slightly below the critical couplings, the light $4 \mathrm{~d}$ mass is given by

$$
p^{2}=m^{2}-\frac{2}{R^{2}} \frac{\mu J_{1}\left(\frac{\mu}{\sqrt{\pi}}\right) /\left(\sqrt{\pi} J_{0}\left(\frac{\mu}{\sqrt{\pi}}\right)\right)}{1-\ln \frac{R}{\epsilon} \mu J_{1}\left(\frac{\mu}{\sqrt{\pi}}\right) /\left(\sqrt{\pi} J_{0}\left(\frac{\mu}{\sqrt{\pi}}\right)\right)} .
$$

Very close to the perturbative critical coupling (21), the light mass becomes

$$
p^{2}=m^{2}-\frac{1}{\pi R^{2}} \frac{\mu^{2}}{1-\frac{\mu^{2}}{2 \pi} \ln \frac{R}{\epsilon}}=m^{2}-\frac{1}{\pi R^{2}} \mu^{2}\left(R^{-1}\right)
$$

and has again a transparent interpretation in terms of the classical running between the compactification scale and the cutoff.

There are also light $4 \mathrm{~d}$ states for $q^{2} \sim R^{-2}$ or larger. However, we are especially interested in the case of small bulk masses $m^{2} R^{2} \ll 1$, for reasons to be explained in the dual NJL formulation of a supersymmetric extension of this model.

\section{$3 \quad$ Symmetry breaking phase transition in orbifolds}

More standard and easy to handle spaces in string theory are orbifolds. We will consider in the following a compactification on the orbifold $T^{2} / \mathbb{Z}_{2}$ and check as a warmup the properties of the phase transition. The orbifold acts as the reflection $\left(y_{1}, y_{2}\right) \rightarrow\left(-y_{1},-y_{2}\right)$. This orbifold has four fixed points. The fixed points and their corresponding $\mathbb{Z}_{2}$ coordinate transformations are summarized as:

$$
\begin{array}{cccc}
y_{1} \rightarrow-y_{1} & y_{1} \rightarrow-y_{1}+2 \pi R_{1} & y_{1} \rightarrow-y_{1} & y_{1} \rightarrow-y_{1}+2 \pi R_{1} \\
y_{2} \rightarrow-y_{2} & y_{2} \rightarrow-y_{2} & y_{2} \rightarrow-y_{2}+2 \pi R_{2} & y_{2} \rightarrow-y_{2}+2 \pi R_{2} \\
(0,0) & \left(\pi R_{1}, 0\right) & \left(0, \pi R_{2}\right) & \left(\pi R_{1}, \pi R_{2}\right) .
\end{array}
$$

In complex notation, the action of $\mathbb{Z}_{2}$ on the compact space is a two-dimensional $\pi$ rotation, $Z_{2}\left(y_{1}+i y_{2}\right)=e^{i \pi}\left(y_{1}+i y_{2}\right)$.

The field equation is free in the bulk and has a delta function source at the origin, suitably replaced by a mass distribution

$$
\partial_{M} \partial^{M} \Phi+\frac{\partial V}{\partial \Phi} \delta^{2}(\mathbf{y})=0 .
$$


Let us now proceed to study the mass spectrum for a scalar field with antiperiodic boundary conditions in the $y_{1}$-direction 6

$$
\Phi\left(y_{1}+2 \pi R_{1}, y_{2}\right)=-\Phi\left(y_{1}, y_{2}\right)
$$

If the scalar field $\Phi$ is even under the orbifold action, it can be decomposed on a complete basis formed by the cosine functions:

$$
\Phi(x, \mathbf{y})=\sum_{\left(k_{1}, k_{2}\right) \in \mathcal{I}} \frac{1}{\sqrt{2 \pi^{2} R_{1} R_{2}}} \cos \left(\frac{k_{1}+1 / 2}{R_{1}} y_{1}+\frac{k_{2}}{R_{2}} y_{2}\right) \phi_{\left(k_{1}, k_{2}\right)}(x) .
$$

The indices $k_{1,2}$ belong to the set $\mathcal{I}$

$$
\mathcal{I}=\{(0 ; 0),(1 \ldots \infty ; 0),(0,-1 ; 1 \ldots \infty),(1 \ldots \infty ; 1 \ldots \infty),(1 \ldots \infty ;-\infty \ldots-1)\}
$$

In the unbroken vacuum, the quadratic part of the scalar action takes the following form after integration over the two extra dimensions

$$
\mathcal{L}=\mathcal{L}_{k i n}-\frac{1}{2} \sum_{\left(k_{1}, k_{2}\right) \in \mathcal{I}}\left(\frac{\left(k_{1}+1 / 2\right)^{2}}{R_{1}^{2}}+\frac{k_{2}^{2}}{R_{2}^{2}}\right) \phi_{\left(k_{1}, k_{2}\right)}^{2}+\bar{\mu}^{2}\left(\sum_{\left(k_{1}, k_{2}\right) \in \mathcal{I}} \phi_{\left(k_{1}, k_{2}\right)}\right)^{2}
$$

where

$$
\bar{\mu}^{2} \equiv \frac{\mu^{2}}{4 \pi^{2} R_{1} R_{2}}
$$

is the naive (volume suppressed) four dimensional lightest scalar mass. The mass term of the $4 \mathrm{~d}$ action is

$$
\mathcal{L}_{\text {mass }}=-\frac{1}{2} \sum_{\left(k_{1}, k_{2}\right),\left(p_{1}, p_{2}\right) \in \mathcal{I}} \phi_{\left(k_{1}, k_{2}\right)} \mathcal{M}_{\left(k_{1}, k_{2}\right),\left(p_{1}, p_{2}\right)}^{2} \phi_{\left(p_{1}, p_{2}\right)}
$$

with the mass matrix given by

$$
\mathcal{M}_{\left(k_{1}, k_{2}\right),\left(p_{1}, p_{2}\right)}^{2}=-2 \bar{\mu}^{2}+\left(\frac{\left(k_{1}+1 / 2\right)^{2}}{R_{1}^{2}}+\frac{k_{2}^{2}}{R_{2}^{2}}\right) \delta_{k_{1}, p_{1}} \delta_{k_{2}, p_{2}}
$$

The diagonalization of this mass matrix defines the physical mass eigenstates.

Let us now try to find the eigenvalues and eigenvectors of the mass matrix (32). We use the techniques used in $5 \mathrm{~d}$ models in [8, 9] and in $6 \mathrm{~d}$ models in [7. The characteristic equation is given by

$$
\mathcal{M}^{2} \Psi_{m}=m^{2} \Psi_{m}
$$

\footnotetext{
${ }^{6}$ This is the analog of the Dirichlet boundary condition on the disk imposed in [2]. As will become clear from our discussion, different boundary conditions, for example $\Phi\left(y_{1}, y_{2}+2 \pi R_{2}\right)=-\Phi\left(y_{1}, y_{2}\right)$ or $\Phi\left(y_{1}+2 \pi R_{1}, y_{2}+2 \pi R_{2}\right)=-\Phi\left(y_{1}, y_{2}\right)$ lead to the same critical coupling (5).
} 
where $m^{2}$ represents the eigenvalues and $\Psi$ is the eigenvector in the basis $\left|k_{1}, k_{2}\right\rangle_{\left(k_{1}, k_{2}\right) \in \mathcal{I}}$, i.e $\Psi_{\left(k_{1}, k_{2}\right)}=\left\langle k_{1}, k_{2} \mid \Psi_{m}\right\rangle$. The matrix equation (33) is equivalent to the infinite set of explicit equations for every $\left(k_{1}, k_{2}\right) \in \mathcal{I}$

$$
2 \bar{\mu}^{2} \Psi^{\prime}=\left(-m^{2}+\frac{\left(k_{1}+1 / 2\right)^{2}}{R_{1}^{2}}+\frac{k_{2}^{2}}{R_{2}^{2}}\right) \Psi_{\left(k_{1}, k_{2}\right)},
$$

where $\Psi^{\prime}$ is independent of $\left(k_{1}, k_{2}\right)$. The solution of the equations (34) is given by

$$
\Psi_{\left(k_{1}, k_{2}\right)}=\frac{\mathcal{N}}{-m^{2}+\left(k_{1}+1 / 2\right)^{2} / R_{1}^{2}+k_{2}^{2} / R_{2}^{2}},
$$

where $\mathcal{N}$ is a normalization constant independent of $\left(k_{1}, k_{2}\right)$. Putting this solution back in the equation (34) and using the fact that

$$
\sum_{\left(k_{1}, k_{2}\right) \in \mathcal{I}}=\frac{1}{2} \sum_{k_{1}, k_{2}=-\infty}^{\infty}
$$

we obtain the eigenvalue equation

$$
\frac{1}{\bar{\mu}^{2}}=\sum_{k_{1}, k_{2}=-\infty}^{\infty} \frac{1}{-m^{2}+\left(k_{1}+1 / 2\right)^{2} / R_{1}^{2}+k_{2}^{2} / R_{2}^{2}},
$$

or equivalently

$$
\begin{aligned}
& \frac{1}{\mu^{2}}=D\left(p^{2}=m^{2}, y_{1}=y_{2}=0\right) \quad, \quad \text { where } \\
& D\left(p^{2}, y_{1}, y_{2}\right)=\frac{1}{4 \pi^{2} R_{1} R_{2}} \sum_{k_{1}, k_{2}=-\infty}^{\infty} \frac{\cos \left[\left(k_{1}+1 / 2\right) y_{1} / R_{1}+k_{2} y_{2} / R_{2}\right]}{-p^{2}+\left(k_{1}+1 / 2\right)^{2} / R_{1}^{2}+k_{2}^{2} / R_{2}^{2}}
\end{aligned}
$$

is the propagator in a mixed, $4 \mathrm{~d}$ momentum and $2 \mathrm{~d}$ position, representation. We want to find an estimate for the lightest solution, $\mathrm{m}^{2}$, of the eigenvalue equation (37). The procedure we are using is similar to the one used in [7] and we only give the result here. It is clear from (37) that there is a critical coupling, defined for arbitrary radii by

$$
\frac{4 \pi^{2} R_{1} R_{2}}{\mu_{c}^{2}}=\sum_{\left|k_{i}\right|<k_{i}^{\max }} \frac{1}{\left(k_{1}+1 / 2\right)^{2} / R_{1}^{2}+k_{2}^{2} / R_{2}^{2}},
$$

which signals a second order phase transition, where the lightest mass $m^{2}$ changes sign. For equal and large radii and by cutting the sums at $k_{i}^{\max }=R \Lambda$, we find $\mu_{c}$ to be exactly equal to the value (5) worked out in [2]. A puzzle arises however in this KK approach to the phase transition. Indeed, whereas we accurately describe the perturbative critical coupling (5), eq. (39) does not contain the nonperturbative couplings $\mu_{c}^{(n)}$ present in (11). We believe that this is due to the way the logarithmic divergence is handled in (39), or equivalently, to the 
"brane resolution" for nonperturbative values of $\mu^{2}$. Indeed, as we already mentioned, in this case wave functions oscillate significantly inside the brane and the regularization procedure becomes more subtle.

Very close and slightly below the phase transition we can linearize the mass equation (37) in order to find for the lightest mode

$$
m^{2} \simeq \frac{4 \pi^{2}}{\alpha R_{1} R_{2}} \frac{\mu_{c}^{2}-\mu^{2}}{\mu_{c}^{4}} \simeq \frac{4 \pi^{2}}{\alpha R_{1} R_{2}}\left(\frac{1}{\mu^{2}}-\frac{1}{\mu_{c}^{2}}\right)
$$

where

$$
\alpha=R_{1}^{-2} R_{2}^{-2} \sum_{k_{1}, k_{2}=-\infty}^{\infty} \frac{1}{\left[\left(k_{1}+1 / 2\right)^{2} / R_{1}^{2}+k_{2}^{2} / R_{2}^{2}\right]^{2}} .
$$

The mass (40) is not exactly the same as the one worked out in [2]. The reason is that the geometries in [2] and in the present section are different and affect the IR physics, in particular physical masses. UV physics, however is the same for both geometries ; in particular the value (at the leading order in $\mu^{2}$ ) of the perturbative critical coupling $\mu_{c}$ defining the phase transition is the same as in (5). Since the regularizations used on the disk and in (39) are different, this shows the regularization independence of the perturbative critical coupling.

Above the critical value, the scalar gets a vev (more precisely, a profile in the compact space)

$$
\phi_{c}\left(y_{1}, y_{2}\right)=\phi_{0} \mathcal{N} \sum_{k_{1}, k_{2}} \frac{\cos \left[\left(k_{1}+1 / 2\right) y_{1} / R_{1}+k_{2} y_{2} / R_{2}\right]}{\left(k_{1}+1 / 2\right)^{2} / R_{1}^{2}+k_{2}^{2} / R_{2}^{2}}
$$

where according to 2

$$
\phi_{0}^{2}=\frac{\mu^{2}-\mu_{c}^{2}}{\lambda}
$$

and $\mathcal{N}$ is a normalization constant such that at the origin (more precisely, at the regularized mass distribution), $\phi_{c}(0,0)=\phi_{0}$.

\section{Localized matter and dual description : the NJL model}

An immediate question is how to generate in a natural way the localized scalar potential needed for the symmetry breaking. The simplest idea is to add (Weyl for definiteness, but the situation is similar for Dirac fermions) $N$ fermions $\chi_{i}$ on the boundary, with Yukawa couplings

$$
g \chi_{i} \chi_{i} \phi(y=0)+\text { h.c. },
$$

to the (now complex) bulk field. The model has, in addition to a global $S O(N)$ symmetry, a continuous chiral $U(1)$ symmetry under which $\phi$ is charged. 


\subsection{The bulk picture}

One-loop quantum corrections generated by the fermion loops automatically generate a scalar potential of the appropriate form (11), plus higher-order terms. The continuous chiral symmetry will now be spontaneously broken at the phase transition.

In the large $N$ limit, the leading induced scalar potential is

$$
V_{\mathrm{eff}}(\phi)=-N \int \frac{d^{4} p}{(2 \pi)^{4}} \ln \left(p^{2}+4 g^{2}|\phi|^{2}\right)
$$

which can be expanded in powers of $\phi$ as

$$
V_{\text {eff }}(\phi)=-4 N g^{2}|\phi|^{2} \int \frac{d^{4} p}{(2 \pi)^{4}} \frac{1}{p^{2}}+8 N g^{4}|\phi|^{4} \int \frac{d^{4} p}{(2 \pi)^{4}} \frac{1}{p^{4}}+\cdots .
$$

We therefore induced radiatively, to the leading order in an $1 / N$ expansion, a potential of the form (11) with

$$
\mu^{2}=\frac{N g^{2}}{4 \pi^{2}} \Lambda^{2}
$$

As usual [10] the power expansion in $\phi$ has severe IR divergences, which are however resummed in the effective potential (45). Then the condition defining the symmetry breaking phase is

$$
\frac{N g^{2}}{4 \pi^{2}} \Lambda^{2}>\frac{4 \pi}{\ln \left(R^{2} \Lambda^{2}\right)}
$$

whereas the perturbative expansion used in [2], for $\mu^{2} \ll 1$, translates here into

$$
\frac{N g^{2}}{4 \pi^{2}} \Lambda^{2} \ll 1
$$

For $\langle\phi\rangle \neq 0$ the brane fermions $\chi_{i}$ acquire a mass and the chiral symmetry is spontaneously broken, with the imaginary part of $\phi$ being the Goldstone boson.

\subsection{Dual picture : the NJL model}

There is a dual description in which the bulk field $\phi$ is integrated out at tree-level and the chiral symmetry breaking is entirely described in terms of nonperturbative brane dynamics. The resulting brane lagrangian has the simple form

$$
S_{\text {brane }}=-i \chi_{i} \sigma^{\mu} \partial_{\mu} \bar{\chi}_{i}+G \chi_{i} \chi_{i} \bar{\chi}_{j} \bar{\chi}_{j}
$$

with

$$
G=\frac{g^{2}}{2 \pi^{2} R_{1} R_{2}} \sum_{\left(k_{1}, k_{2}\right) \in \mathcal{I}} \frac{1}{\frac{\left(k_{1}+1 / 2\right)^{2}}{R_{1}^{2}}+\frac{k_{2}^{2}}{R_{2}^{2}}} \simeq \frac{g^{2}}{4 \pi} \ln \left(R^{2} \Lambda^{2}\right),
$$

where in the last equality we considered equal and large radii $R_{1}=R_{2}=R \gg M_{*}^{-1}$, where $M_{*}$ is the $6 \mathrm{~d}$ fundamental (Planck) scale. Therefore the "dual" lagrangian (50) is the 
Nambu-Jona-Lasinio model [3], in which the chiral symmetry is dynamically broken by the fermion condensate $\left\langle\chi_{i} \chi_{i}\right\rangle$ for values of the four-fermion coupling above the critical value

$$
G>G_{c} \quad, \quad \text { where } G_{c}^{-1}=\frac{N \Lambda^{2}}{4 \pi^{2}} .
$$

By using (47) and (51), we find that the condition (52) is precisely the same as the condition for the broken phase derived in the "bulk" approach (48).

Whereas in the deep IR the $6 \mathrm{~d}$ bulk model is equivalent to the $4 \mathrm{~d}$ NJL model, their UV behaviour is different ${ }^{7}$. In particular, due to the cumulative effects of the KK states, the four-fermion coupling $G$ has a logarithmic running

$$
G(Q) \simeq \frac{g^{2}}{4 \pi} \ln \left(\Lambda^{2} / Q^{2}\right)
$$

from the cutoff scale $\Lambda$ to the compactification scale $R^{-1}$, as illustrated in Fig.(11). So $G$ increases in the IR and can generate dynamical chiral symmetry breaking. Even for couplings $g^{2} \ll 4 \pi$ such that a perturbative treatment is available, the non-decoupling of heavy KK states generates a large four-fermion coupling in the infrared which drives the symmetry breaking.

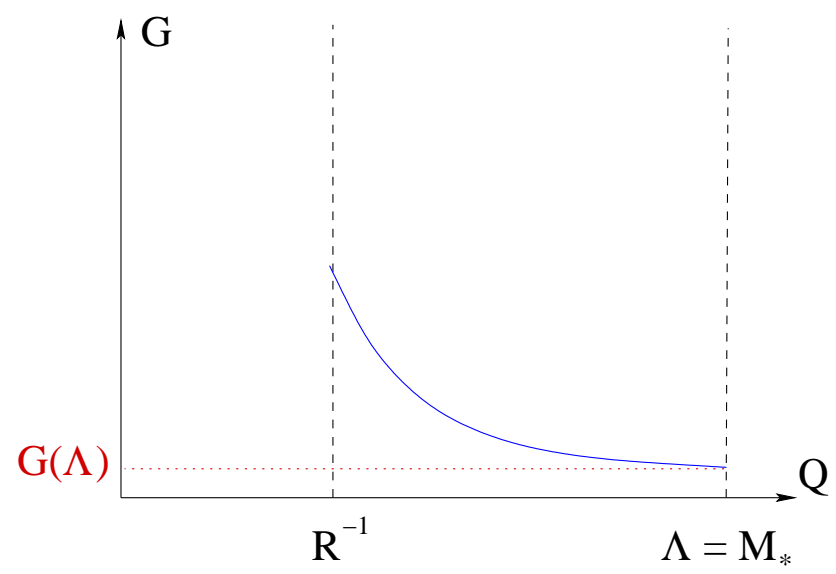

Figure 1: Classical running of the four-fermion coupling induced by the cumulative effect of the KaluzaKlein states. The coupling becomes strong $G=G_{c}$ at the compactification scale and induces chiral symmetry breaking.

In the bulk formulation, the mass parameter was generated through quantum loops and the phase transition had a "classical" (quantum mechanical) nature. In the NJL formulation,

\footnotetext{
${ }^{7}$ For earlier ideas of the role of extra dimensions in dynamical symmetry breaking, see [11. For a recent extensive review on strong dynamics, see e.g. [22].
} 
the four-fermion coupling is generated classically while the phase transition is generated in a nonperturbative fashion through the quantum gap equation

$$
1=4 N G \int \frac{d^{4} p}{(2 \pi)^{4}} \frac{1}{p^{2}+m^{2}}=4 N G D\left(x=0, m^{2}\right) \text {. }
$$

In the previous expression, $D\left(x, m^{2}\right)$ is the $4 \mathrm{~d}$ propagator in the position representation of a scalar field, and $m$ is the dynamical fermion mass.

The counterpart of the NJL quantum gap equation (154) in the bulk formulation is the classical gap equation (37)-(38). The similarity of the bulk equation (38) and the NJL gap equation (54) is transparent.

Notice that the dual NJL description is valid in principle only below the phase transition. In this case and when the classical running interpetation is valid, the connection between the Higgs-localized scalar potential and the NJL model can also be understood in terms of the compositeness condition of [5]. There, it was argued, by introducing a composite scalar field $H$, that the kinetic term $Z_{H}|\partial H|^{2}$ vanishes, $Z_{H} \rightarrow 0$, at the compositeness scale $\Lambda_{C}$. By a rescaling of the kinetic term to the canonical form, this is equivalent of imposing the boundary conditions $m_{H} \rightarrow \infty, \lambda_{H} \rightarrow \infty$, where the parameters $m_{H}, \lambda_{H}$ are defined from the effective scalar potential $V=-m_{H}^{2}\left|H^{2}\right|+\lambda_{H}|H|^{4}$. In our $6 \mathrm{~d}$ model, the running between the UV cutoff and the compactification scale $R^{-1}$ produces $\mu^{2}$ and $\lambda$ to diverge at $R^{-1}$ precisely at the critical point. With a rescaling $\phi \rightarrow Z^{1 / 2} \phi$ of the scalar to go from the convention of [2] to the normalization of [5], keeping the scalar mass fixed, the wave function is

$$
Z(Q)=1-\frac{\mu^{2}}{2 \pi} \ln \frac{\Lambda}{Q}
$$

At the phase transition $\mu=\mu_{c}$, we get

$$
Z\left(Q=R^{-1}, \mu=\mu_{c}\right)=0 \quad, \quad \lambda\left(Q=R^{-1}\right)=\lambda(\Lambda) Z^{-2}\left(Q=R^{-1}, \mu=\mu_{c}\right) \rightarrow \infty .
$$

The first of these conditions is similar to the one-loop (large $\mathrm{N}$ ) induced wave function displayed in [5], whereas the one for scalar self-coupling is different, since the UV physics is different. A natural candidate for the compositeness scale in our case is therefore $\Lambda_{C}=1 / R$.

Analogously to the scalar parameters $\mu, \lambda$, the Yukawa coupling $g$ gets an induced running which can be easily integrated :

$$
Q \frac{d g}{d Q}=-\frac{\mu^{2}}{2 \pi} g \quad, \quad g(Q)=\frac{g(\Lambda)}{1-\frac{\mu^{2}}{2 \pi} \ln \frac{\Lambda}{Q}} .
$$

The compositeness conditions have therefore to be supplemented with

$$
g\left(Q=R^{-1}\right) \rightarrow \infty
$$

in analogy with the compositeness condition for the top quark in the top condensation scenario [5]. Whereas the first and the third conditions (56)-(58) are indeed similar to the 
compositeness conditions in [5], close to the critical coupling $\mu_{c}$ the running interpretation breaks down and the $4 \mathrm{~d}$ physics is actually completely perturbative. Indeed, defining the $4 \mathrm{~d}$ effective theory as in [2] by

$$
\begin{array}{ll}
\phi\left(x^{\mu}, r\right)=\sqrt{\frac{2}{\pi R^{2}}} \ln \frac{R}{\epsilon} \sigma\left(x^{\mu}\right), \quad r<\epsilon, \\
\phi\left(x^{\mu}, r\right)=\sqrt{\frac{2}{\pi R^{2}}} \ln \frac{R}{r} \sigma\left(x^{\mu}\right), \quad & \epsilon<r<R,
\end{array}
$$

we find, at $\mu \simeq \mu_{c}$, the $4 \mathrm{~d}$ scalar self-coupling and Yukawa coupling to the fermions to be

$$
\lambda_{4}=\frac{64 \pi^{2}}{\mu_{c}^{8}} \frac{\lambda}{R^{4}} \quad, \quad g_{4}=\frac{2 \sqrt{2 \pi}}{\mu_{c}^{2}} \frac{g}{R} .
$$

Since $1 / \mu_{c}^{2} \sim \ln (\Lambda R)$, the couplings (60) are log enhanced compared to their naive (volume suppressed) values, but are still perturbative and under control.

Whereas the NJL 4d description and the bulk $6 \mathrm{~d}$ one with appropriate boundary conditions are equivalent in the IR, the $6 \mathrm{~d}$ picture can sometimes be simpler to use in order to describe the symmetry breaking pattern. For example, consider two set of fermions living in two different fixed points, $(0,0)$ and $\left(0, \pi R_{2}\right)$ and interacting both with (relatively large) Yukawa coupling to the same 6d bulk scalar, which has nontrivial boundary conditions (26)). In the NJL picture, there are three four-fermionic interactions coming from the two sets of fermions, with specific four-fermion couplings. In the bulk picture, localized loops of fermions generate localized scalar potentials at both fixed points. The scalar action in this case is

$$
\begin{aligned}
& S=\int d^{4} x d^{2} y\left[\frac{1}{2}\left(\partial_{M} \phi\right)^{2}-V_{\delta, 1}(\phi)-V_{\delta, 2}(\phi)\right] \\
& V_{\delta, 1}(\phi)=\left(-\frac{\mu_{1}^{2}}{2} \phi^{2}+\frac{\lambda_{1}}{4} \phi^{4}\right) \cdot \delta\left(y_{1}\right) \delta\left(y_{2}\right), \\
& V_{\delta, 2}(\phi)=\left(-\frac{\mu_{2}^{2}}{2} \phi^{2}+\frac{\lambda_{2}}{4} \phi^{4}\right) \cdot \delta\left(y_{1}\right) \delta\left(y_{2}-\pi R_{2}\right) .
\end{aligned}
$$

It is a straightforward exercise to work out the equation defining the mass eigenstates (333) in the unbroken phase. We find

$$
\begin{aligned}
& {\left[\frac{1}{\bar{\mu}_{1}^{2}}-\sum_{k_{i}=-\infty}^{\infty} \frac{1}{m^{2}+\left(k_{1}+1 / 2\right)^{2} / R_{1}^{2}+k_{2}^{2} / R_{2}^{2}}\right] \times\left[\frac{1}{\bar{\mu}_{2}^{2}}-\sum_{k_{i}=-\infty}^{\infty} \frac{1}{m^{2}+\left(k_{1}+1 / 2\right)^{2} / R_{1}^{2}+k_{2}^{2} / R_{2}^{2}}\right]} \\
& =\left(\sum_{k_{i}=-\infty}^{\infty} \frac{(-1)^{k_{2}}}{m^{2}+\left(k_{1}+1 / 2\right)^{2} / R_{1}^{2}+k_{2}^{2} / R_{2}^{2}}\right)^{2} .
\end{aligned}
$$

Putting different fermions in different positions in the compact space can also provide a geometrical understanding of various values of their Yukawa couplings via the wave function profile of the bulk scalar. 


\section{$5 \quad$ Supersymmetric extension and coupling to localized chiral fields}

The basic mechanism we used for generating a sizable running uses the logarithmic terms coming from the renormalization of the localized mass term. This implies in particular that the corrections to the mass should be forbidden in the bulk. The natural way to implement this is to have supersymmetry in the bulk. Since the potential has to be generated on the brane, we add boundary chiral fields in supersymmetric multiplets, with supersymmetry softly broken on the boundary.

There are two inequivalent ways to supersymmetrize, by using either bulk hypermultiplets or bulk vector multiplets. Let us start with the first case and consider a bulk hypermultiplet in $6 \mathrm{~d}$, which in $4 \mathrm{~d} \mathcal{N}=1$ supersymmetric language has two chiral (super)fields $\Phi_{1,2}=$ $\left(\phi_{1,2}, \psi_{1,2}\right)$, and $N$ localized matter superfields $A_{i}=\left(z_{i}, \chi_{i}\right)$, with $i=1 \cdots N$, where we denoted in parenthesis the scalar and fermionic components of the multiplets. By using the $\mathcal{N}=1$ superfield formalism of Ref. [12, the action is given by

$$
\begin{aligned}
S= & \int d^{2} y d^{4} \theta\left(\Phi_{1}^{\dagger} \Phi_{1}+\Phi_{2}^{\dagger} \Phi_{2}\right)+\left(\int d^{2} y d^{2} \theta \Phi_{1}\left(\partial_{5}+i \partial_{6}\right) \Phi_{2}+\text { h.c. }\right) \\
& +\int d^{4} \theta A_{i}^{\dagger} A_{i}\left(1-\Sigma^{2} \theta^{2} \bar{\theta}^{2}\right)+\left(\int d^{2} \theta g \Phi_{1}(y=0) A_{i} A_{i}+\text { h.c. }\right)
\end{aligned}
$$

where $\Sigma$ is a scalar soft mass term for the boundary fields. In order to write the component lagrangian, we first solve for the auxiliary fields

$$
\begin{aligned}
& F_{\phi, 1}^{\dagger}=-\left(\partial_{5}+i \partial_{6}\right) \phi_{2}-g z_{i} z_{i} \delta^{2}(y), \\
& F_{\phi, 2}^{\dagger}=\left(\partial_{5}+i \partial_{6}\right) \phi_{1} \quad, \quad F_{A_{i}}^{\dagger}=-2 g \phi_{1} z_{i} .
\end{aligned}
$$

After eliminating auxiliary fields, the component lagrangian is

$$
\begin{aligned}
S= & \int d^{2} y\left[\left|\partial_{M} \phi_{1}\right|^{2}+\left|\partial_{M} \phi_{2}\right|^{2}-i \psi_{1} \sigma^{\mu} \partial_{\mu} \bar{\psi}_{1}-i \psi_{2} \sigma^{\mu} \partial_{\mu} \bar{\psi}_{2}+\left(\psi_{1}\left(\partial_{5}+i \partial_{6}\right) \psi_{2}+\text { h.c. }\right)\right] \\
& -\left[S_{\text {kin }}\left(z_{i}, \chi_{i}\right)+g \phi_{1} \chi_{i} \chi_{i}+2 g z_{i} \psi_{1} \chi_{i}+4 g^{2}\left|\phi_{1}\right|^{2}\left|z_{i}\right|^{2}\right. \\
& \left.+g\left\{z_{i} z_{i}\left(\partial_{5}-i \partial_{6}\right) \bar{\phi}_{2}+\text { h.c. }\right\}+\Sigma^{2}\left|z_{i}\right|^{2}+g^{2} z_{i}^{2} \bar{z}_{j}^{2} \delta^{2}(0)\right]\left.\right|_{y=0} .
\end{aligned}
$$

Analogously to the non supersymmetric case we impose nontrivial boundary conditions

$$
\Phi_{1,2}\left(y_{1}+2 \pi R_{1}, y_{2}\right)=-\Phi_{1,2}\left(y_{1}, y_{2}\right) .
$$

The $\mathbb{Z}_{2}$ orbifold has a nontrivial action on the bulk hypermultiplet fields. A consistent assignement is $\Phi_{1}$ to be even and $\Phi_{2}$ to be odd. The KK expansions in this case are

$$
\begin{aligned}
& \Phi_{1}(x, \mathbf{y})=\sum_{\left(k_{1}, k_{2}\right) \in \mathcal{I}} \frac{1}{\sqrt{2 \pi^{2} R_{1} R_{2}}} \cos \left(\frac{k_{1}+1 / 2}{R_{1}} y_{1}+\frac{k_{2}}{R_{2}} y_{2}\right) \Phi_{1}^{\left(k_{1}, k_{2}\right)}(x), \\
& \Phi_{2}(x, \mathbf{y})=\sum_{\left(k_{1}, k_{2}\right) \in \mathcal{I}} \frac{1}{\sqrt{2 \pi^{2} R_{1} R_{2}}} \sin \left(\frac{k_{1}+1 / 2}{R_{1}} y_{1}+\frac{k_{2}}{R_{2}} y_{2}\right) \Phi_{2}^{\left(k_{1}, k_{2}\right)}(x) .
\end{aligned}
$$


The second possibility of supersymmetrization is to add bulk $6 \mathrm{~d}$ vector multiplets, which in $4 \mathrm{~d} \mathcal{N}=1$ language are described by vector $V$ and chiral $\phi$ supermultiplets, both in the adjoint representation of a gauge group $G$. In order to be able to couple $\phi$ to the localized matter, we need to choose $Z_{2}$, the orbifold action, such that $V$ is odd and therefore has no zero modes, whereas $\phi$ is even and can therefore couple to boundary chiral multiplets. More precisely, we can start from a nonabelian gauge group and give a nontrivial action of the orbifold on the gauge degrees of freedom

$$
V\left(-y_{1},-y_{2}\right)=P^{\dagger} V\left(y_{1}, y_{2}\right) P \quad, \quad \phi\left(-y_{1},-y_{2}\right)=-P^{\dagger} \phi\left(y_{1}, y_{2}\right) P,
$$

where $P$ is a matrix in the adjoint representation of the gauge group, such that $P^{2}=1$. The surviving (even) gauge group generators $T_{a}$ satisfy $\left[T_{a}, P\right]=0$, whereas the remaining ones $T_{\alpha}$ are projected out. The complementary states $\phi_{\alpha}$ from the adjoint scalar $\phi$ survive at the fixed points and can consistently be coupled to the localized matter. By imposing nontrivial boundary conditions on $\phi_{\alpha}$ we generate a setup where $\phi_{\alpha}$ plays the role of the scalar field with localized Higgs potential and can trigger the phase transition. While this can be an interesting alternative, we will not pursue this possibility further on.

\subsection{Bulk picture}

The leading quantum corrections in $1 / N$ come at one-loop with the chiral (super)fields $A_{i}$ running in the loop. There is an induced effective potential for $\phi_{1}$ which can be computed in the standard way [10]. The result is

$$
V_{\mathrm{eff}}\left(\phi_{1}\right)=N \int \frac{d^{4} p}{(2 \pi)^{4}}\left\{\ln \left(p^{2}+\Sigma^{2}+4 g^{2}\left|\phi_{1}\right|^{2}\right)-\ln \left(p^{2}+4 g^{2}\left|\phi_{1}\right|^{2}\right)\right\}
$$

which can be expanded in powers of $\phi_{1}$ as

$V_{\mathrm{eff}}\left(\phi_{1}\right)=-4 N \Sigma^{2} g^{2}\left|\phi_{1}\right|^{2} \int \frac{d^{4} p}{(2 \pi)^{4}} \frac{1}{p^{2}\left(p^{2}+\Sigma^{2}\right)}+8 N \Sigma^{2} g^{4}\left|\phi_{1}\right|^{4} \int \frac{d^{4} p}{(2 \pi)^{4}} \frac{2 p^{2}+\Sigma^{2}}{p^{4}\left(p^{2}+\Sigma^{2}\right)^{2}}+\cdots$.

We therefore induced radiatively, in the leading order in an $1 / N$ expansion, a potential of the form (11) with

$$
\mu^{2}=\frac{N g^{2}}{4 \pi^{2}} \Sigma^{2} \ln \frac{\Lambda^{2}}{\Sigma^{2}}
$$

In a first approximation, we can consider this as the bare coupling in the model of [2]. Then the condition defining the symmetry breaking phase, for large and equal radii $R_{1}=R_{2} \gg \frac{1}{\Lambda}$, is

$$
\frac{N g^{2}}{4 \pi^{2}} \Sigma^{2} \ln \frac{\Lambda^{2}}{\Sigma^{2}}>\frac{4 \pi}{\ln \left(R^{2} \Lambda^{2}\right)},
$$

whereas the perturbativity condition translates here into

$$
\frac{N g^{2}}{4 \pi^{2}} \Sigma^{2} \ln \frac{\Lambda^{2}}{\Sigma^{2}} \ll 1
$$


For $\left\langle\phi_{1}\right\rangle \neq 0$ the brane fermions $\chi_{i}$ acquire a mass and the chiral symmetry is spontaneously broken. Finally, fermion loops also induce localized operators of the form $\left|\left(\partial_{5}+i \partial_{6}\right) \phi_{2}\right|^{2}$. Their effect is to renormalize the KK masses of the odd field $\phi_{2}$, but this effect has no relevance for our present discussion.

Notice that for natural values of (the dimensionful coupling) $g$, eq. (72) can be satisfied only for large values of the soft breaking parameter $\Sigma$. This is easy to interpret in the dual NJL description, to which we now turn.

\subsection{Dual description : the softly supersymmetric NJL model}

There is a dual description in which the bulk fields $\Phi_{i}$ are integrated out at tree-level and the chiral symmetry breaking is entirely described in terms of nonperturbative brane dynamics. Since the bulk plus the interaction action is supersymmetric, the integrating out procedure gives rise to a supersymmetric effective action, to add to the brane lagrangian with softly broken supersymmetry. There are some subtleties in proving that the integration out leads to a well-defined four-dimensional action without ill-defined (i.e. $\left.\delta^{2}(0)\right)$ terms. Analogously to former studies in $5 \mathrm{~d}$ [13], it can be checked that the singular terms cancel out as they should. The resulting brane lagrangian has the simple form

$$
S_{\text {brane }}=\int d^{4} \theta\left\{A_{i}^{\dagger} A_{i}\left(1-\Sigma^{2} \theta^{2} \bar{\theta}^{2}\right)+G A_{i} A_{i} A_{j}^{\dagger} A_{j}^{\dagger}\right\},
$$

where

$$
G=\frac{g^{2}}{2 \pi^{2} R_{1} R_{2}} \sum_{\left(k_{1}, k_{2}\right) \in \mathcal{I}} \frac{1}{\frac{\left(k_{1}+1 / 2\right)^{2}}{R_{1}^{2}}+\frac{k_{2}^{2}}{R_{2}^{2}}} .
$$

Therefore the "dual" lagrangian is a softly-broken supersymmetric version [14, 15] of the Nambu-Jona-Lasinio model [3]. The dynamics of the softly broken supersymmetric version of the NJL model in the large $N$ expansion was investigated in detail in [15]. It was found there that chiral symmetry is dynamically broken by the fermion condensate $\left\langle\chi_{i} \chi_{i}\right\rangle$, for values of the four-fermion coupling above the critical value

$$
G>G_{c} \quad, \quad \text { where } G_{c}^{-1}=\frac{N \Sigma^{2}}{4 \pi^{2}} \ln \frac{\Lambda^{2}}{\Sigma^{2}} .
$$

By using (75), we find that the condition (76) is precisely the same as the condition for the broken phase derived in the previous section, which for equal and large radii is displayed in (72).

As in the $4 \mathrm{~d}$ supersymmetric NJL model, there is a naturalness problem in the $6 \mathrm{~d}$ construction. In the 4d SUSY NJL model, the symmetry breaking occurs for values of the four-fermion interaction $G \gtrsim 1 / \Sigma^{2} \gg 1 / \Lambda^{2}$ much larger than its natural value. For small supersymmetry breaking $\Sigma R \ll 1$, this generates strong four-fermion interactions well below the compositeness scale $\Lambda_{c}=R^{-1}$. As transparent in (75), the natural scale of the strong four-fermion interactions for our $6 \mathrm{~d}$ explicit realization is actually $R^{-1}$, unless $g$ is much larger than its natural value. The reason is simpler to understand by rewriting (744) in an 
appropriate form to compare with the Minimal Supersymmetric Standard Model (MSSM) 14

$$
\begin{aligned}
S_{\text {brane }}= & \int d^{4} \theta\left\{A_{i}^{\dagger} A_{i}\left(1-\Sigma^{2} \theta^{2} \bar{\theta}^{2}\right)+H_{1}^{\dagger} H_{1}\right\}+ \\
& \left(\int d^{2} \theta H_{2}\left(m H_{1}-g A_{i} A_{i}\right)+\text { h.c. }\right) .
\end{aligned}
$$

Since $H_{2}$ is a Lagrange multiplier in (77), the two lagrangians (74) and (77) are equivalent for $G=g^{2} / m^{2}$. As explained in [14, 15], $H_{2}$ acquires a kinetic term $Z_{2} H_{2}^{\dagger} H_{2}$ at one-loop in the large $N$ expansion, which vanishes at the compositeness scale $Z_{2}\left(Q=\Lambda_{c}\right)=0$. Below $\Lambda_{c}$, both $H_{1}$ and $H_{2}$ are dynamical fields and are to be identified with the two Higgs doublets of the MSSM. In the language of (177), the naturalness problem is that in order to keep the Higgs mass light, the supersymmetric mass term should be of order $m \sim \Sigma$, which is nothing but the reincarnation of the so-called $\mu$-problem of MSSM in the SUSY NJL case. In the 6d case, the analog of the action (177) is (63), the analog of $H_{2}$ is $\Phi_{1}$, whereas the analog of the supersymmetric mass $m$ is the mixing term $\Phi_{1}\left(\partial_{5}+i \partial_{6}\right) \Phi_{2}$ in (63). Upon KK expansion, we get $m \sim\left(1 / 2 R_{1}\right)$. Equivalently, this can be seen from the nontrivial boundary conditions (66) via the KK expansion (67). Since the symmetry breaking only occurs for $m \sim \Sigma$, the model also requires large supersymmetry breaking scale, as already anticipated.

If we believe in the equivalence between the $6 \mathrm{~d}$ scalar model and the $4 \mathrm{~d}$ NJL model also close to the nonperturbative critical couplings of section 2, then the corresponding Yukawa coupling $g$ can be large and produce a larger Fermi coupling $G$. However it will be hard to argue reliably for very large Yukawas. Maybe a simpler road is to use a small bulk mass $m R \ll 1$, which will generate a large Fermi coupling $G \sim g^{2} / R^{2} m^{2}$. Another solution in order to get symmetry breaking compatible with small supersymmetry breaking scale $\Sigma$ is to start with small supersymmetry mass generated by boundary conditions. In other words, we need

$$
\left(\Phi_{1}+i \Phi_{2}\right)\left(y_{1}+2 \pi R_{1}, y_{2}\right)=e^{2 \pi i \omega}\left(\Phi_{1}+i \Phi_{2}\right)\left(y_{1}, y_{2}\right)
$$

with $\omega \ll 1$, in which case $m \sim\left(\omega / R_{1}\right)$. Whereas from first principles in string theory $\omega$ is quantized and cannot be very small, in analogy with known 5 d examples $[9, \omega \ll 1$ can actually be realized by starting with periodic boundary conditions and adding small supersymmetric mass terms for bulk fields localized at the fixed points $\left(\pi R_{1}, 0\right)$ and/or $\left(\pi R_{1}, \pi R_{2}\right)$. Supersymmetry is broken softly with $\Sigma \Lambda \ll 1$ only at the origin $(0,0)$. After re-diagonalization of the mass matrix, this is equivalent to starting with nontrivial boundary conditions (78) and no localized mass terms. This is technically natural in the sense that a small supersymmetric mass term in the fixed points is protected by supersymmetry.

\section{String theory realization}

It is legitimate to ask if it is possible to realize the field theory construction we did provide in [2] and in this paper from a string theory framework. The answer is positive and we 
provide here one possible construction for the (softly broken) supersymmetric case of section $5^{8}$. The main requirement for the string construction is to provide a large and flat twodimensional compact space, so there should be no localized source which would curve the large $2 \mathrm{~d}$ space. Indeed, our field theory analysis was done in flat space. This situation can be realized in orientifold constructions, where orientifold planes cancel the sources provided by the branes. The basic ingredients of the construction are that the field $\phi$ arises from D5 branes wrapping our large $2 \mathrm{~d}$ space, whereas the localized fermions arise at the intersection between the D5 branes and a different set of branes, the intersection being four dimensional and generating chiral fermions. We use orientifolds of type IIB string theory with D5-branes wrapping different coordinates of the internal space. Our example is based on the orientifold projection $\Pi^{\prime}=\Pi \pi_{4} \pi_{5} \pi_{8} \pi_{9}$, where $\Pi$ is the left-right world-sheet interchange, $\pi_{4}, \pi_{5}$ are parity operations in two compact coordinates $x_{4}$ and $x_{5}$, to be identified with the two large dimensions $\left(y_{1}, y_{2}\right)$ in our field theoretical construction and $\pi_{8}, \pi_{9}$ are parity operations in two internal noncompact coordinates $x_{8}$ and $x_{9}$. The basic building block for brane configuration we consider is then the following

$\begin{array}{lllllllllll}\text { coord. } & 0 & 1 & 2 & 3 & 4 & 5 & 6 & 7 & 8 & 9 \\ \mathrm{D}_{1} & \mathrm{x} & \mathrm{x} & \mathrm{x} & \mathrm{x} & \mathrm{x} & \mathrm{x} & 0 & 0 & 0 & 0 \\ \mathrm{D} 5 & \mathrm{x} & \mathrm{x} & \mathrm{x} & \mathrm{x} & 0 & 0 & \mathrm{x} & \mathrm{x} & 0 & 0 \\ \mathrm{O} 5_{2} & \mathrm{x} & \mathrm{x} & \mathrm{x} & \mathrm{x} & 0 & 0 & \mathrm{x} & \mathrm{x} & 0 & 0\end{array}$

In (79), crosses $\mathrm{x}$ denote coordinates parallel to the branes, whereas 0 denotes orthogonal coordinates. Notice that $\mathrm{D} 5_{2}$ branes and $\mathrm{O} 5_{2}$ planes are orthogonal to the large $2 \mathrm{~d}$ space $\left(x_{4}, x_{5}\right)$. In order to keep the $2 \mathrm{~d}$ space flat we need a configuration with $\mathrm{D} 5_{2}$ branes on top of the $\mathrm{O} 5_{2}$ planes, with locally zero tension and charge. We add a Wilson line $\left\langle W_{4}\right\rangle$ on the D $5_{1}$ branes in the compact $x_{4}$ coordinate, which has the effect of breaking the gauge group and giving masses to fields charged under $W_{4}$. There are in particular four charged scalar fields $\phi_{i}$, which get a mass from this Hosotani mechanism and will be identified with the master field(s) of our field theory model. Notice first of all that this field lives indeed in six dimensions, in the bulk of our large $2 \mathrm{~d}$ compact space and it corresponds to a hypermultiplet $\phi=\left(\phi_{1}, \phi_{2}\right)$ from the $4 \mathrm{~d}$ viewpoint, as in section 5. The mass of $\phi$ is positive and it corresponds to the mass generated by boundary conditions analyzed in [2] and in sections 2, 4 and 5 of the present paper. Alternatively, using a Wilson line in one of the last four coordinates $x_{6} \cdots x_{9}$ is equivalent to considering a bulk supersymmetric mass as in the section 3. For simplicity, we can consider the last three coordinates $x_{7} \cdots x_{9}$ as being noncompact, whereas $x_{6}$ is a circle and will be used to break supersymmetry a la Scherk-Schwarz.

The $\mathrm{D} 5_{2}$ branes gauge fields are nondynamical in four dimensions and play the role of global symmetries. The D5 1 brane degrees of freedom, on the other hand, are dynamical and contain in particular gauge fields and the field(s) $\phi$. The $\mathrm{D} 55_{1}-\mathrm{D} 5_{2}$ sector, after additional orbifold projections to be discussed below, contains massless $\mathcal{N}=1$ chiral multiplets localized

\footnotetext{
${ }^{8}$ Other realizations of dynamical symmetry breaking can be found in [16 for string realizations of a nonlocal NJL version and [17 ([1] ) for a string (field-theory) realization of the chiral symmetry breaking in QCD.
} 
in four dimensions, to be identified with the $4 \mathrm{~d}$ chiral multiplets $A_{i}$ in section 5 . At the effective low energy action level, the setup is similar to the one considered in section 5 , with couplings of the form $A \phi A$ and one expects the arguments presented there to apply and generate dynamical symmetry breaking. Non-trivial boundary conditions in the compact coordinate $x_{6}$ a la Scherk-Schwarz break supersymmetry at tree-level in the D5 2 sector, whereas the $D 5_{1}$ branes, being orthogonal to the $x_{6}$ coordinate, feel the breaking only through radiative corrections [19]. Notice that the dynamics in the large bulk coordinates $x_{4}, x_{5}$ is supersymmetric at that stage. At one-loop, supersymmetry breaking propagates in the $\mathrm{D} 5_{1^{-}}$ $\mathrm{D} 5_{2}$ sector and generates the localized $4 \mathrm{~d}$ soft terms that were used in section 5 .

The setup preserve until now $\mathcal{N}=2$ supersymmetry in $4 \mathrm{~d}$ spontaneously broken to $\mathcal{N}=0$ by the Scherk-Schwarz deformation, so additional ingredients are needed in order to generate chirality. The standard internal spaces used in this respect are the Calabi-Yau spaces or the orbifolds. We choose here the second possibility. We introduce additional $Z_{2}$ and $Z_{3}$ orbifold operations acting on the internal coordinates as

$$
\begin{aligned}
& Z_{2}\left(z_{1}, z_{2}, z_{3}\right)=\left(-z_{1},-z_{2}, z_{3}\right) \quad, \quad Z_{3}\left(z_{1}, z_{2}, z_{3}\right)=\left(e^{\frac{2 \pi i}{3}} z_{1}, z_{2}, e^{-\frac{2 \pi i}{3}} z_{3}\right) \\
& \text { where } \quad z_{1}=\frac{x_{4}+i x_{5}}{\sqrt{2}}, \quad z_{2}=\frac{x_{6}+i x_{7}}{\sqrt{2}}, \quad z_{3}=\frac{x_{8}+i x_{9}}{\sqrt{2}}
\end{aligned}
$$

are the three complex internal coordinates. The resulting orientifold, which is dual to the so-called $Z_{3} \times Z_{2}$ or $Z_{6}^{\prime}$ type I orbifold in the literature [20] after performing four T-dualities in $x_{6}, x_{7}, x_{8}, x_{9}$, reduces supersymmetry down to $\mathcal{N}=1$ in $4 \mathrm{~d}$. The $4 \mathrm{~d}$ type $\mathrm{I} Z_{3} \times Z_{2}$ orbifold has D9 brane / O9 planes and one set of D5 branes / O5 planes, wrapping the third internal torus. After the four T-dualities, the D9 branes (O9 planes) become our D 51 branes (O5 planes) and the D5 (O5) branes become our D5 2 branes ( $5_{2}$ planes). Our Wilson line is in the type I orbifold a Wilson line on the D9 branes.

In order to break completely supersymmetry, as already announced we are adding a Scherk-Schwarz operation in the compact coordinate $x_{6}$, compatible with the two orbifold operations. Our Scherk-Schwarz deformation is a $2 \pi$ rotation in $x_{6}$ and one $4 \mathrm{~d}$ spacetime coordinate. The corresponding worldsheet current anticommutes with the $Z_{2}$ orbifold projection and commutes with the $Z_{3}$ one, as required by the consistency of the string construction [21. A last subtlety, explained in detail in [19] is that due to the Scherk-Schwarz operation, the $\mathrm{O} 5_{1}$ planes, which are perpendicular to the $x_{6}$ coordinate used in the supersymmetry breaking, are actually pairs of $\mathrm{O} 5_{1}$ orientifold $-\bar{O} 5_{1}$ antiorientifold planes, situated at $x_{6}=0$ and $x_{6}=\pi R_{6}$, respectively. If the radius $R_{6} \gg l_{s}$ is large enough, the closed string tachyon is massive and the timescale for the instability can be large enough. The D $5_{1}$ branes should be at (or close to) the point $x_{6}=0$, such that the strings D $5_{1}-\bar{O} 5_{1}$, which break supersymmetry, to be very massive.

A possible objection to the present setup is that the boundary conditions generated mass and/or the bulk hypermultiplet mass $m$ are not constant but field-dependent, given by the vev of the Wilson line on the D $5{ }_{1}$ branes. Since the setup in non-supersymmetric, the Wilson line acquires a potential and its vev will be dynamically fixed. This last point needs further investigation which is however beyond the goals of the present paper. This objection is also 
valid for the field-theory construction, in that we assumed that the radii of the large $2 \mathrm{~d}$ space were stabilized by additional dynamics. To conclude, the setup presented in this section does realize the (softly) supersymmetric model work out in section 5, where the supersymmetry breaking is soft and comes from a Scherk-Schwarz deformation in an extra space coordinate.

The original non-superymmetric setup of [2] and in sections 2,3 of the present paper can be in principle also realized for smaller values of $R_{6} \gtrsim l_{s}$. However in this case we expect severe tachyonic instabilities in the system, which need to be suppressed in order for the string picture to be a viable description of the field-theory construction.

Acknowledgements : We thank T. Gherghetta, A. Pomarol and V. Rubakov for useful discussions. E.D. thanks the Galileo Institute of Theoretical Physics and INFN for partial support during the completion of this work. Work partially supported by the CNRS PICS \# 2530 and 3059, RTN contracts MRTN-CT-2004-005104 and MRTN-CT-2004-503369 and the European Union Excellence Grant, MEXT-CT-2003-509661.

\section{References}

[1] The literature on gravitational aspects of codimension two models is vast. Some references are A. G. Cohen and D. B. Kaplan, Phys. Lett. B 470 (1999) 52 arXiv:hep-th/9910132; T. Gherghetta and M. E. Shaposhnikov, Phys. Rev. Lett. 85 (2000) 240 |arXiv:hep-th/0004014|; I. Navarro, JCAP 0309 (2003) 004 arXiv:hep-th/0302129; Y. Aghababaie, C. P. Burgess, S. L. Parameswaran and F. Quevedo, Nucl. Phys. B 680 (2004) 389 arXiv:hep-th/0304256; G. W. Gibbons, R. Guven and C. N. Pope, Phys. Lett. B 595 (2004) 498 arXiv:hep-th/0307238; M. Peloso, L. Sorbo and G. Tasinato Phys. Rev. D 73 (2006) 104025 arXiv:hep-th/0603026.

[2] E. Dudas, C. Papineau and V. A. Rubakov, JHEP 0603 (2006) 085 arXiv:hep-th/0512276.

[3] Y. Nambu and G. Jona-Lasinio, Phys. Rev. 122 (1961) 345.

[4] Y. Nambu, EFI-88-62-CHICAGO Invited talk to appear in Proc. of 1988 Int. Workshop New Trends in Strong Coupling Gauge Theories, Nagoya, Japan, Aug 24-27, 1988; V. A. Miransky, M. Tanabashi and K. Yamawaki, Phys. Lett. B 221 (1989) 177.

[5] W. A. Bardeen, C. T. Hill and M. Lindner, Phys. Rev. D 41 (1990) 1647.

[6] W. D. Goldberger and M. B. Wise, Phys. Rev. D 65 (2002) 025011 arXiv:hep-th/0104170; K. A. Milton, S. D. Odintsov and S. Zerbini, Phys. Rev. D 65 (2002) 065012 arXiv:hep-th/0110051|; F. del Aguila, M. Perez-Victoria and J. Santiago, JHEP 0302 (2003) 051 |arXiv:hep-th/0302023.

[7] E. Dudas, C. Grojean and S. K. Vempati, arXiv:hep-ph/0511001. 
[8] K. R. Dienes, E. Dudas and T. Gherghetta, Phys. Rev. D 62 (2000) 105023 arXiv:hep-ph/9912455.

[9] J. Bagger, F. Feruglio and F. Zwirner, JHEP 0202 (2002) 010 arXiv:hep-th/0108010.

[10] S. R. Coleman and E. Weinberg, Phys. Rev. D 7 (1973) 1888.

[11] B. A. Dobrescu, Phys. Lett. B 461 (1999) 99 arXiv:hep-ph/9812349; N. ArkaniHamed, H. C. Cheng, B. A. Dobrescu and L. J. Hall, Phys. Rev. D 62 (2000) 096006 arXiv:hep-ph/0006238; M. Hashimoto, M. Tanabashi and K. Yamawaki, Phys. Rev. D 64 (2001) 056003 arXiv:hep-ph/0010260. V. Gusynin, M. Hashimoto, M. Tanabashi and K. Yamawaki, Phys. Rev. D 65 (2002) 116008 arXiv:hep-ph/0201106.

[12] N. Marcus, A. Sagnotti and W. Siegel, Nucl. Phys. B 224 (1983) 159; N. Arkani-Hamed, T. Gregoire and J. G. Wacker, JHEP 0203 (2002) 055 arXiv:hep-th/0101233.

[13] E. A. Mirabelli and M. E. Peskin, Phys. Rev. D 58 (1998) 065002 arXiv:hep-th/9712214; E. Dudas, T. Gherghetta and S. Groot Nibbelink, Phys. Rev. D 70 (2004) 086012 arXiv:hep-th/0404094.

[14] W. Buchmuller and S. T. Love, Nucl. Phys. B 204 (1982) 213; W. Buchmuller and U. Ellwanger, Nucl. Phys. B 245 (1984) 237.

[15] T. E. Clark, S. T. Love and W. A. Bardeen, Phys. Lett. B 237 (1990) 235; M. Carena, T. E. Clark, C. E. M. Wagner, W. A. Bardeen and K. Sasaki, Nucl. Phys. B 369 (1992) 33; P. Binetruy, E. A. Dudas and F. Pillon, Nucl. Phys. B 415 (1994) 175 arXiv:hep-ph/9304278.

[16] E. Antonyan, J. A. Harvey, S. Jensen and D. Kutasov, arXiv:hep-th/0604017; A. Parnachev and D. A. Sahakyan, arXiv:hep-th/0604173.

[17] M. Kruczenski, D. Mateos, R. C. Myers and D. J. Winters, JHEP 0405 (2004) 041 arXiv:hep-th/0311270; T. Sakai and S. Sugimoto, Prog. Theor. Phys. 113 (2005) 843 arXiv:hep-th/0412141.

[18] J. Erlich, E. Katz, D. T. Son and M. A. Stephanov, Phys. Rev. Lett. 95 (2005) 261602 arXiv:hep-ph/0501128; L. Da Rold and A. Pomarol, Nucl. Phys. B 721 (2005) 79 arXiv:hep-ph/0501218.

[19] I. Antoniadis, E. Dudas and A. Sagnotti, Nucl. Phys. B 544 (1999) 469 arXiv:hep-th/9807011; I. Antoniadis, G. D'Appollonio, E. Dudas and A. Sagnotti, Nucl. Phys. B 553 (1999) 133 arXiv:hep-th/9812118.

[20] G. Zwart, Nucl. Phys. B 526 (1998) 378 arXiv:hep-th/9708040; G. Aldazabal, A. Font, L. E. Ibanez and G. Violero, Nucl. Phys. B 536 (1998) 29 arXiv:hep-th/9804026. 
[21] R. Rohm, Nucl. Phys. B 237 (1984) 553; S. Ferrara, C. Kounnas, M. Porrati and F. Zwirner, Nucl. Phys. B 318 (1989) 75.

[22] C. T. Hill and E. H. Simmons, Phys. Rept. 381 (2003) 235 [Erratum-ibid. 390 (2004) 553] arXiv:hep-ph/0203079. 